\title{
L'appel nominal, une technique pour la démocratie extrême (1789-1795) ?
}

Is the "appel nominal" a technique for radical democracy (1789-1795)?

Anne Simonin et Corinne Lechevanton-Gomez

\section{(2) OpenEdition \\ 12 Journals}

Édition électronique

URL : https://journals.openedition.org/ahrf/10555

DOI : 10.4000/ahrf.10555

ISSN : 1952-403X

Éditeur :

Armand Colin, Société des études robespierristes

Édition imprimée

Date de publication : 1 septembre 2009

Pagination : 67-101

ISBN : 978-2-200-92559-8

ISSN : 0003-4436

Référence électronique

Anne Simonin et Corinne Lechevanton-Gomez, "L'appel nominal, une technique pour la démocratie extrême (1789-1795) ? », Annales historiques de la Révolution française [En ligne], 357 | juillet-septembre 2009, mis en ligne le 01 septembre 2012, consulté le 23 avril 2022. URL : http:// journals.openedition.org/ahrf/10555; DOI : https://doi.org/10.4000/ahrf.10555 


\title{
L'APPEL NOMINAL, UNE TECHNIQUE POUR LA DÉMOCRATIE EXTRÊME (1789-1795) ?
}

Anne SIMONIN

avec la collaboration de Corinne LECHEVANTON-GOMEZ

\begin{abstract}
L'appel nominal est une technique de vote en vigueur dans les assemblées révolutionnaires dès 1789 . Cette manière de recueillir les voix des députés lors des votes critiques a été considérée par les historiens de la Législative et de la Convention comme le plus sûr moyen d'analyser les forces politiques en présence. Mais l'appel nominal peut aussi être pensé comme une technique politique, emportant avec elle une théorie originale de la représentation. Cette manière de voter permet aussi une définition matérielle du Centre, identifié à ceux qui votent ni oui ni non. Grâce à l'appel nominal, la minorité législative conquiert son droit d'expression : elle ne menace pas la majorité qui sort d'un vote dont les résultats ne sont pas douteux. L'appel nominal, qui eût pu servir à modérer des conflits irréductibles en favorisant l'expression de la minorité pendant la législature tout en légitimant le point de vue de la majorité, disparaît de façon révélatrice sous la Terreur, comme cela apparaît clairement dans les listes des quarante-six appels nominaux recensés entre 1789 et 1795 (listes consultables sur le site internet : http://ihrf.univparis1.fr Rubrique : "les outils").
\end{abstract}

Mots-clés : listes d'infamie, machines à voter, François-Élie Guirault, abstentions, centre, minorité, représentation.

(1) Nous remercions Jean-Philippe Heurtin de sa lecture attentive et de ses suggestions. 
L'appel nominal, dit aussi « appel nominatif », est une technique de vote en vigueur dans les assemblées révolutionnaires ${ }^{2}$ : à l'appel de son nom par l'un des secrétaires de l'assemblée, chaque député se lève et répond « présent », s'il s'agit d'un appel nominal de contrôle, « oui » ou « non » si une question lui est posée. Sujet aride, l'appel nominal est aussi la source de nombreuses anecdotes qui colorent la vie parlementaire de la Révolution.

Vote public, l'appel nominal peut être motivé à la tribune : lors de l'un des appels nominaux concernant la peine à appliquer au roi Louis XVI, le 16 janvier 1793, on vit les députés défiler à la barre de la Convention pendant vingt-quatre heures ${ }^{3}$. La tension extrême qui régnait alors dans l'assemblée faillit faire une victime en la personne du secrétaire Manuel qui « venait de quitter la salle ; un tumulte effroyable s'élève dans l'Assemblée ; on entend crier et on répète de tous côtés : Arrêtez Manuel! arrêtez le ! Duhem rentre aussitôt avec Manuel, qui apprend qu'on croyait qu'il emportait les listes de l'appel nominal », rapporte Léonard Gallois ${ }^{4}$.

Le 13 avril 1793, l'appel nominal ayant pour objet le décret d'accusation contre Marat mobilisa la Convention pendant neuf heures : « Marat est avec Louis XVI le seul personnage de la Révolution a avoir paru digne d'un appel nominal, et le seul donc sur lequel on dispose, commentaires appropriés à l'appui, des votes de toute la Convention $»^{5}$. À ceci près que Marat ne fut pas le seul député à être traité avec les égards d'un roi, le représentant du peuple Carrier, lui aussi mis en accusation fit, le 3 frimaire an III (23 novembre 1794), l'objet d'un appel nominal également motivé. Cambacérès prévoyait le pire : « On motivera son opinion, comme dans l'affaire de Marat et de Capet, et tout le monde sait que ces appels nominaux durent vingt-quatre heures. Pendant ce temps le gouvernement ne marche point ; car les membres de la Convention, étant obligés d'assister à la séance, ne [peuvent] pas être dans les comités $[\ldots] »^{6}$. L'appel nominal concernant Carrier ne dura qu'un peu plus de sept heures

(2) L'appel nominal est la technique de vote obligatoire dans les assemblées primaires. Voir Serge ABERDAm et alii, Voter, élire pendant la Révolution française 1789-1799: guide pour la recherche, CTHS, 2006, p. 542.

(3) Voir Tableau $\mathrm{n}^{\circ}$ 3-3: Recension des appels nominaux de la Convention.

(4) Léonard Gallois, Histoire de la Convention nationale par elle-même, Chez Auguste Mie, 1834, t. II, p. 349-350.

(5) Mona Ozouf, « Marat », dans François Furet, Mona Ozouf (dir.), Dictionnaire critique de la Révolution française. Acteurs, Flammarion, coll. «Champs », 1992, p. 180.

(6) Archives parlementaires, t. CII, 2 frimaire an III (22 novembre 1794), p. 25. 
(annexe $\left.3^{7}\right) \ldots$ Singuliers, les "grands» appels nominaux devaient demeurer l'exception, tout en faisant l'objet d'une exploitation intensive de la part des historiens disposant, grâce à eux, de données objectives quant à la nature des votes et leurs motivations.

En prenant appui «sur les six appels nominaux de 1793 » (procès du roi, accusation contre Marat et affaire de la Commission des Douze), dans une étude magistrale publiée au début des années soixante-dix, The Men of the First French Republic. Political Alignments in the National Convention of 1792, Alison Patrick délimitait les trois grandes tendances politiques de la Convention, la Montagne, la Plaine, la Gironde, en précisant les appartenances des députés ${ }^{8}$. L'idée n'était peut-être pas neuve : n'était-ce pas, comme le faisait remarquer Françoise Brunel, Georges Pariset qui, dès 1920, avait attiré l'attention des historiens sur l'utilité des appels nominaux pour l'histoire politique de la première Ière République, celle d'avant la Terreur ${ }^{9}$ ? Reste qu'elle n'avait jamais été exploitée avec cette ampleur. Ni d'ailleurs suscitée si intense controverse : au terme de son étude, Alison Patrick remettait en question les résultats auxquels était parvenu Michael J. Sydenham dans son ouvrage pionnier sur Les Girondins ${ }^{10}$ : elle donnait corps, et nombre ${ }^{11}$, à ce qui selon Sydenham n'était que stratégies discursives d'une «minorité agressive » de trublions, les Montagnards qui, en vue de disqualifier leurs adversaires... les qualifiaient de 《 girondins $»^{12}$. Le heurt des interprétations recouvrait des options politiques différentes ${ }^{13}$, mais posait également des questions de méthode qui

(7) Consultable sur : www.ihrf.univ-paris1.fr. Rubrique "les outils".

(8) Alison Patrick, The Men of the First French Republic. Political Alignments in the National Convention of 1792, Baltimore, The Johns Hopkins University Press, 1972, p. 6 et 83-136.

(9) Françoise BRunEL, « Images et approches des Montagnards. Une histoire de la Montagne est-elle possible? », dans Michel Vovelle (dir.), L'Image de la Révolution française, 1989, vol. I, p. 506.

(10) Michael J. Sydenham, The Girondins, Londres, The Athlon Press, 1961.

(11) Les girondins rassemblent 178 députés, soit 23,8\% de la Convention ; les Montagnards représentent 302 députés, soit 40,3\% de la Convention; la Plaine, 250 députés soit 33,4 \% de la Convention, dans Alison PATRICK, The Men of the First French Republic, op. cit., p. 30.

(12) Alison PATRICK, « Political Divisions in the French National Convention », The Journal of Modern History, vol. 41, n 4, dec. 1969, p. 421-422. Michael J. Sydenham lui répondra : «The Montagnards and Their Opponents : Some Considerations on a Recent Reassessment of the Conflicts in the French National Convention 1792-1793 », The Journal of Modern History, vol. 43, $\mathrm{n}^{\circ} 2$, jun. 1971 , p. 421-422

(13) Alison Patrick « reproduit ce qu'on pourrait appeler la version jacobine autorisée. Sans le savoir peut-être, Sydenham, lui, fournit une contribution importante, et l'une des premières à un courant qui répudie la version jacobine dans son ensemble [...] », Michael S. Lewis-Beck, Anne Hildreth et Alan B. Sitzer, «Y a-t-il eu un groupe girondin à la Convention nationale (17921793) ? », dans François Furet et Mona Ozouf (dir.), La Gironde et les Girondins, Payot, 1989, p. 170. 
excédaient les convictions respectives des auteurs. Pouvait-on, ainsi que le faisait Alison Patrick, plaquer sur les résultats des appels nominaux une grille anachronique, celle du groupe politique préexistant (Montagne, Plaine, Gironde) à l'engagement de chaque député ? L'analyse exclusive de certains appels nominaux de 1793 encourait également le reproche, Alison Patrick ne l'ignorait pas ${ }^{14}$, d'être une analyse statique ne permettant pas d'avoir une vision d'ensemble des forces politiques des assemblées révolutionnaires avant le mois de janvier 1793 - ce qui était pour le moins tardif.

Passionné par l'histoire de l'assemblée législative, C.J. Mitchell devait, au début des années quatre-vingt, à la fois réaffirmer l'intérêt des historiens pour les appels nominaux et renouveler leur approche théorique. Il suggérait, en effet, d'abandonner la distribution des députés entre des groupes structurés au profit d'une «nouvelle terminologie inhérente aux appels nominaux $\rangle^{15}$ : les radicaux ou « votants-oui » et les conservateurs ou « votants-non ». Le grand perdant de cette analyse était le centre qui s'évanouissait ${ }^{16}$. C.J. Mitchell devait toutefois convenir, quelques années plus tard, que cette notion de centre était fondamentale pour comprendre la géographie politique de la Législative ; Pierre Serna en démontrerait la complexité philosophico-politique en forgeant la notion d' ' extrême centre $»^{17}$. Et si l'appel nominal participait aussi de la philosophie, de la pratique et de la définition du centre en temps d'exception, permettant d'identifier au centre ceux qui se trouvent littéralement sous la pression des deux côtés de l'Assemblée, et ne votent ni oui, ni non ${ }^{18}$ ? Analyser le Centre ou la Plaine en se fondant sur le vote des ni pour, ni contre permettrait ainsi de rompre avec la vision réprobatrice de l'historiographie révolutionnaire justement dénoncée par Michel Biard : le

\footnotetext{
(15) C.J. Mitchell, The French Legislative Assembly of 1791, New York, E. J. Brill, 1988, p. $19-20$.

(16) C.J. Mitchell, « Political Divisions within the Legislative Assembly of 1791 », French Historical Studies, vol. 13, $\mathrm{n}^{\circ}$ 3, Spring 1984, p. 358 et p. 365. Voir aussi The French Legislative Assembly of 1791, op.cit., p. 14-25;286-295.

(17) Pierre SERnA, «Existe-t-il un extrême centre? Le point aveugle de la République directoriale. L'exemple de La Décade », dans Jacques Guilhaumou et Raymonde MonNier (dir.), Des notions-concepts en révolution autour de la liberté politique à la fin du XVIII siècle, Paris, SER, 2003, p. 154 ; « Barère, penseur et acteur d'un premier opportunisme républicain face au Directoire exécutif », $A H R F, \mathrm{n}^{\circ} 332$, avril-juin 2003, p. 106 et La République des girouettes. 1789-1815 et audelà, la France de l'extrême centre, Seyssel, Champ Vallon, 2005, p. 350-356.
} p. 423.

(14) Alison Patrick, «Political Divisions in the French National Convention », art. cité,

(18) C.J. MitchelL, The French Legislative Assembly of 1791, op. cit., p. 18. 
Centre est tout autre chose qu'une " mouvance informelle de députés indécis, voire veules, qui se sont contentés d'apporter leurs suffrages aux uns et aux autres en fonction des évolutions politiques $\gg{ }^{19}$. Le Centre gagnerait à être étudié à partir de ceux qui, lors de scrutins à haute charge politique décidés par appel nominal, ont publiquement choisi de ne pas choisir, et n'ont donc voté ni en faveur du oui, ni en faveur du non.

Sous la Révolution, dans les assemblées parlementaires, le vote a lieu habituellement "par assis et levé »: " Que ceux qui sont d'avis que telle chose ait lieu se lèvent » dit le président. Si la majorité est douteuse, il engage une seconde épreuve : "Que ceux qui sont d'avis contraire se lèvent $»^{20}$. Et si le doute subsiste, alors intervient l'appel nominal.

Sans être le mode de votation le plus fréquent, l'appel nominal est toutefois la façon de voter règlementaire en cas de doute sur la majorité dégagée par assis ou levés (tableau $\mathrm{n}^{\circ} 1-1$ ). Inscrit dans le règlement des assemblées depuis 1789, l'appel nominal, organisé pour la première fois les 9 et 10 mai 1789, a tendance à se généraliser, donc à se banaliser ainsi que l'attestent les appels nominaux qui rythment la vie de la Constituante. Technique régulière d'élection du président et des secrétaires, l'appel nominal, à partir de septembre 1792, aura lieu tous les quinze jours ${ }^{21}$ (tableau $\mathrm{n}^{\circ} 1-3$ ).

À côté de ces appels nominaux ordinaires - qui n'apparaissent pas dans les tableaux de l'annexe 2 -, existent des appels nominaux qui, pour des raisons diverses, n'ont pas débouché sur un vote : soit, comme le 24 novembre 1791, que le nombre des députés requis pour la tenue des délibérations (200), ait été atteint avant la fin de l'appel nominal ${ }^{22}$; soit, comme le 27 mai 1793, que la parole demandée par Robespierre afin de protester contre l'arrestation d'Hébert et de Varlet ordonnée par la Commission des Douze, soit devenue sans objet: "Tout à coup un bruit considérable se fait entendre à l'une des deux portes de la salle; on se

(19) Michel BiARD, «Entre Gironde et Montagne. Les positions de la Plaine au sein de la Convention nationale au printemps 1793 », Revue historique, vol. 3, n 631, 2004, p. 573.

(20) M. DE L'ÉPITHÈTE, Dictionnaire national et anecdotique, À Politicopolis, Chez les marchands de nouveauté, 1790, article : «Épreuve », p. 76-77.

(21) Art. 1 à 5 du Règlement du 28 septembre 1792, dans Roger BonnARD, Les Règlements des assemblées législatives de la France depuis 1789, Paris, Sirey, 1926, p. 146.

(22) Voir Tableau 2-2 et chap. II, art. $1^{\text {er }}$ du règlement de l'assemblée Constituante, dans Roger BonnARD, Les Règlements..., op. cit., p. 120. Cette disposition, non reprise par le règlement de l'assemblée Législative, est visiblement cependant toujours en vigueur. 
plaint que les consignes sont violées et qu'une multitude de citoyens, répandus dans les couloirs obstruent toutes les avenues de la Convention et empêchent toute circulation. - L'appel nominal est interrompu $»^{23}$. On n'aura garde d'oublier à côté des appels nominaux inaboutis, ceux qui n'ont finalement pas eu lieu, celui demandé par le Montagnard Lecointre, le 12 germinal an III ( $1^{\text {er }}$ avril 1795), pour s'opposer à la déportation de Barère, Collot d'Herbois, Billaud-Varennes et de Vadier : la Convention décidera l'impression, «l'envoi aux départemens et aux armées » de la Déclaration et demande en appel nominal et des noms des cinquante-trois députés signataires, livrant les «derniers Montagnards » à l'opprobre d'une opinion publique invitée à voir dans cette action collective «le dernier effort d'une minorité rebelle $\gg^{24} \ldots$ Les appels nominaux interrompus, ceux qui n'ont pas eu lieu, au moins autant que les appels nominaux effectifs, recèlent des possibilités d'interprétation politique inédite des luttes au sein des assemblées révolutionnaires. Ce n'est toutefois pas l'objet de cet article.

Nous ne prétendons pas, en effet, faire ici l'histoire détaillée des quarante-six appels nominaux recensés en annexe 2 - la liste est incomplète malgré les multiples recoupements effectués, mais nous attacher à l'étude de l'appel nominal au singulier, en ce qu'il a de plus sec et de moins historique, en tant que technique régulatrice de deux instances qui, dans le cadre de la «centralité législative » (Françoise Brunel) mise en place par le gouvernement révolutionnaire à l'automne 1793, restent, malgré de nombreux travaux, « un point aveugle de la mémoire et de l'historiographie » révolutionnaires : « Le fonctionnement de l'assemblée et le règlement de la parole publique $»^{25}$. La pratique de l'appel nominal a contribué à définir la fonction de député et à imposer une théorie origi-

(23) Archives parlementaires, séance du 27 mai 1793, t. 65, p. 382.

(24) L'expression est de Bourdon de l'Oise, Le Moniteur, n 195, 15 germinal an III (4 avril 1795), p. 118. Voir aussi Françoise BRUNEL, «Images et approches des Montagnards », art. cité, p. 506 et « Une extrême minorité ne prouve pas le tort... : sur quelques « derniers Montagnards » ou l'an II en l'an III », dans Christine Peyrard (dir.), Minorités politiques en Révolution 1789-1799, Publications de l'Université de Provence, 2007, p. 63-79. Le texte de la « Déclaration et demande en appel nominal faite par plusieurs membres » et de « la loi qui ordonne l'impression de la Déclaration » est au Bulletin des Lois, à la date du 12 germinal an III.

(25) Jean-Claude BonNET, « La sainte masure, sanctuaire de la parole fondatrice », dans $L a$ Carmagnole des muses. L'homme de lettres et l'artiste dans la Révolution, Paris, A. Colin, 1989, p. 198. Lire à ce sujet Jean-Philippe Heurtin, «Architectures morales de l'Assemblée nationale », dans L'Espace public parlementaire. Essai sur les raisons du législateur, Paris, PUF, 1999, p. 63-107. 
nale de la représentation abandonnée lors de la mise en place du gouvernement révolutionnaire à l'automne 1793. Si, au lieu de disparaître, l'appel nominal avait perduré sous la Terreur, le tribut payé par la représentation nationale à la guillotine eût-il été si élevé ?

L'appel nominal est bien davantage qu'un mode règlementaire de recueil des voix en cas de doute sur la majorité : par l'autodiscipline qu'il impose aux députés, et le recours à une infamie de fait pour les contrevenants, l'appel nominal accélère la professionnalisation de la fonction de député sous la Convention ${ }^{26}$. Dans la mesure où il répartit les députés entre votants-oui et votants-non mais tient aussi compte des abstentionnistes, les ni oui, ni non, l'appel nominal permet à la fois d'étudier les courants politiques en ne forçant pas les apparentements, mais aussi de tenter l'étude de ce qui est le plus difficile à fixer, le plus évanescent, le plus complexe à interpréter : le centre, cette « Plaine » qu'Alison Patrick évaluait au tiers des députés sous la Convention (33,4\%). L'appel nominal fait ainsi surgir une question essentielle concernant le fonctionnement démocratique de la République : celle de « la minorité politique en Révolution $»^{27}$. Tout en renforçant le principe majoritaire, en substituant à la «majorité douteuse » dégagée par assis et levés la proclamation de la victoire incontestable d'un camp, l'appel nominal confère une existence institutionnelle à tous ceux qui, selon les catégories de l'époque, n'ont « point de voix ». Il reconnaît ainsi les droits de la minorité, l'autorisant à faire entendre une opinion dissidente qui ne menace pas l'expression de la volonté générale puisqu'elle ne remet pas en cause la décision de la majorité.

Mais, du fait de son insuffisante codification, exploité à des fins politiques, l'appel nominal contribue à entretenir les tensions politiques, voire à les aggraver en obligeant les députés à se distribuer en deux camps irréductibles, les oui et les non. Les deux tentatives de codification de l'appel nominal, l'une sous la Constituante, l'autre sous la Convention, ont tourné court. Elles n'en sont pas moins intéressantes à étudier en ce qu'elles dessinent la possibilité d'une "démocratie extrême » tolérant l'abstention, donc la neutralité, et reconnaissant, à la minorité, des droits.

Les vertus modératrices de l'appel nominal, les Girondins ne les ont pas comprises. Ils les ont même combattues. Ils pourraient bien avoir

(26) Philip Dawson, Provincial Magistrates and Revolutionary Politics in France 17891795, Cambridge, Harvard University Press, 1972, p. 277.

(27) Christine Peyrard, « Avant-propos », dans Minorités politiques en Révolution, op. cit., p. 7-9. 
payé cette incompréhension là de leur vie. Mais plus inattendu : pourquoi les Montagnards proches de Danton, qui, tel Legendre, étaient passés maître dans l'usage de l'appel nominal, n'ont-ils pas demandé que, le 11 germinal an II (31 mars 1794), le décret de mise en accusation de Danton et des députés arrêtés avec lui la nuit précédente, fut voté à l'appel nominal ? On l'ignore. Mais on a la preuve que l'appel nominal était ce que craignaient le plus certains membres du Comité de Salut public, tel Couthon : pourquoi ?

\section{Une technique aux origines floues}

Quelles sont les origines de l'appel nominal ? Il est difficile, voire impossible de répondre avec certitude à cette question. Dans son article classique, «Les origines religieuses des techniques électorales et délibératives modernes », si Léo Moulin atteste la pratique du vote «par assis et levé » dans les communautés religieuses italiennes dès le XIII ${ }^{\mathrm{e}}$ sì̀cle ${ }^{28}$, il ne mentionne pas l'appel nominal. En revanche, «le principe du scrutin secret », allant jusqu'à brûler les bulletins afin d'effacer toute trace des opinions particulières une fois dégagée l'opinion majoritaire est, lui, « incontestablement d'origine religieuse $»^{29}$. Ce qui ne milite pas en faveur des origines religieuses de l'appel nominal, vote public, où l'individu dévoile et son identité et son opinion particulière.

Si l'appel nominal n'a pas retenu l'attention de Léo Moulin, cela ne signifie pas que cette technique de vote n'a pas une origine religieuse. Olivier Christin mentionne un ouvrage de 1683, La Manière de tenir le Chapitre général de l'ordre de Cisteaux où est précisément décrit «le vote par tête » qui pourrait être considéré comme l'ancêtre de l'appel nominal : «Opiner par tête [...] n'est autre chose que donner chacun son suffrage, comme on en fait dans toutes les Compagnies Ecclesiastiques et Laïques dans les Conciles et dans les Parlemens etc. on écoute chacun en particulier, et après que tous ont dit leurs sentimens, on recueille les voix

(28) Léo Moulin, «Les origines religieuses des techniques électorales et délibératives modernes » [1953], Politix. Revue des sciences sociales du politique, vol. 11, n 43, 1998, p. 125 (consultable sur : http ://www.persee.fr). Voir aussi Olivier Christin, « À quoi sert de voter au XVI ${ }^{\mathrm{e}}$ XVIII ${ }^{\mathrm{e}}$ siècle ? ", Actes de la Recherche en Sciences Sociales, ${ }^{\circ} 140,2001 / 4$, p. 27. Je remercie Benoist Pierre des précisions fournies et de m'avoir signalé cet article.

(29) Léo Moulin, « Les origines religieuses... », art. cité, p. 158. Voir aussi l'article « Élection » dans R. NAZ, Dictionnaire de droit canonique, Librairie Letouzey et Ané, 1953, t. V, p. 242243. 
et l'on conclut à la pluralité [majorité] ${ }^{30}$. N'est-ce pas là un appel nominal?

Retraçant dans un tableau « le déroulement des opérations électorales » en 1789 dans les assemblées primaires ${ }^{31}$, Patrice Gueniffey montre que l'appel nominal ne porte pas atteinte au secret du vote. En effet, après la « proclamation du résultat» de l'appel, les secrétaires s'attachent à la «destruction des bulletins et des listes de recensement $»^{32}$. Quant à la pratique des assemblées parlementaires, elle est elle aussi très soucieuse du secret, et préserve l'anonymat des représentants du peuple par le biais d'un mode de décompte original des voix qui ne permet pas de connaître la nature du vote de tel ou tel député, puisque les votes ne sont pas «pointés », enregistrés face au nom du député, mais représentés par des bâtonnets sous la Constituante (A). Une motion déposée le 8 décembre 1789 , et ambitionnant de mettre en face de chaque nom, le résultat du vote, devait être ajournée, confirmant «la répugnance qu'a tôt montré l'Assemblée pour toute forme de publicité des votes $»^{33}$. Cette « répugnance » devait perdurer sous la Législative où les appels nominaux sont décomptés par des chiffres, donc toujours en occultant le nom des députés (B).

(30) Dom Louis Meschet, La Manière de tenir le Chapitre général de l'ordre de Cisteaux, Paris, F. Léonard, 1683, p. 101 et Olivier Christin, « À quoi sert de voter au XVI"-XVIII siècle ? ", art. cité, p. 24. On aboutit ici, en mobilisant des sources différentes, à une conclusion identique à celle de Patrice Gueniffey, Le Nombre et la raison. La Révolution française et les élections, Paris, Éditions de l'EHESS, 1993, p. 283.

(31) Patrice Gueniffey, Le Nombre et la raison, op. cit., p. 274.

(32) Ibid.

(33) André CAstaldo, Les Méthodes de travail de la Constituante, Léviathan, 1989, p. 350. La motion du 8 décembre 1789, contrairement à ce qu'écrit l'auteur, n'a pas été adoptée. Voir ProcèsVerbal de l'Assemblée nationale, Chez Baudouin, t. 5, 1789, n 145 : «Qu'il soit dorénavant procédé à l'appel nominal par liste de noms des membres, dont chaque secrétaire aura un exemplaire sur lequel il notera le oui et le non, de manière que par la confrontation de ces listes, l'on puisse reconnaître où se trouvent les différences et, par là, obtenir un moyen de redresser [les erreurs] ». Cette motion a été ajournée. 
Illustration $\mathrm{n}^{\circ} 1$ : Compter les voix de l'appel nominal A-séance du 5 janvier 1790, sur deux pages séparées ${ }^{34}$

\begin{tabular}{|l|l|}
\hline Oui & Non \\
\hline I I I I I / I I I I I / I I I I I/ I I I I I/ ... & I I I I I / I I I I I / I I I I I/ I I I I I/ ... \\
\hline$\ldots .$. I I I I I / I I I I I / I I I I I/ I I I I I/ & $\ldots$. I I I I I / I I I I I / I I I I I/ I I I I I/ \\
\hline $\begin{array}{l}\text { « 71 fois cinq ou } 5 \text { fois 71 voix } \\
355 \text { voix » }\end{array}$ & $\begin{array}{l}\text { « cinq fois 91 ou 91 fois } 5 \\
455 \text { voix » }\end{array}$ \\
\hline
\end{tabular}

B-séance du 13 avril 1792, sur une seule page, deux colonnes ${ }^{35}$

\begin{tabular}{|c|c|c|c|c|c|}
\hline \multicolumn{3}{|l|}{ Oui } & \multicolumn{3}{|l|}{ Non } \\
\hline 0 & \multicolumn{2}{|l|}{$1.2 .3 .4 .5 \cdot 6.7 .8 .9 .10$} & 0 & \multicolumn{2}{|c|}{1.2 .3 .4 .5 .6 .7 .8 .9 .10} \\
\hline 1 & \multicolumn{2}{|l|}{ 1.2.3.4.5.6.7.8.9.10 } & 1 & \multicolumn{2}{|c|}{ 1.2.3.4.5.6.7.8.9.10 } \\
\hline $2 \ldots$ & \multicolumn{2}{|l|}{ 1.2.3.4.5.6.7.8.9.10 } & $2 \ldots$ & \multicolumn{2}{|c|}{ 1.2.3.4.5.6.7.8.9.10 } \\
\hline $30 \ldots$ & 1.2.3.4.5. & 305 & $23 \ldots$ & 1.2 .3 .4 .5 .6 & $\begin{array}{l}\text { Non } \\
236 \\
\text { Oui } \\
305 \\
=541\end{array}$ \\
\hline 40 & & & 40 & & \\
\hline
\end{tabular}

Ce serait donc sous la Convention, en vue d'influer sur les résultats du scrutin ou d'étouffer toute forme d'opposition, que le vote serait devenu public, et l'appel nominal une dangereuse machine politique, permettant de connaître, diffuser, exploiter... le vote d'un représentant du peuple sur telle ou telle question. À l'appui de cette thèse, l'exemple suivant, qui atteste, au printemps 1793, une véritable révolution dans le décompte des voix, désormais recueillies par les secrétaires sur des feuilles imprimées et nominatives : 
C-séance du 28 mai 1793, imprimé : Liste des citoyens-députés

à la Convention Nationale, 20 p. ${ }^{36}$

«Appel nominal sur le rapport du débat qui supprime

la Commission des Douze avant son rapport»

\begin{tabular}{|c|c|c|c|c|}
\hline Départemens & Oui & Non & Absent & $\begin{array}{l}\text { Absent } \\
\text { blanc* }^{*}\end{array}$ \\
\hline 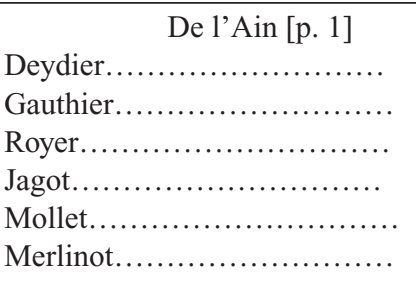 & o & $\begin{array}{l}n \\
n \\
n\end{array}$ & & $\begin{array}{l}12 \text { noms } \\
\text { de députés }\end{array}$ \\
\hline 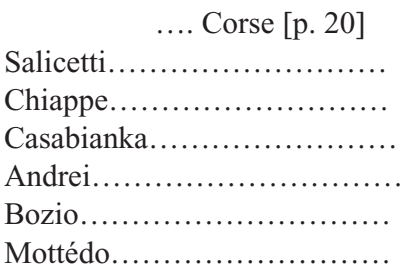 & o & & & \\
\hline & {$[3]$} & {$[3]$} & & \\
\hline
\end{tabular}

*Cette colonne n'est pas imprimée. Elle est de la main du secrétaire qui reporte les votes et n'apparaît qu'à la hauteur du département du Morbihan. Le total des voix est fait par colonne, sur chaque page. Sur la dernière page, figure une addition de deux chiffres sans autre précision : 238 sur 279 avec 517 comme total. Tableau $n^{\circ} 2-3$.

Or, l'appel nominal est devenu un mode de publicité des votes bien avant la Convention, dès la Constituante ainsi que l'attestent les listes de députés qui circulent sous le manteau (annexe 2).

Selon le député du Dauphiné, Jean-Joseph Mounier, c'est le 17 juin 1789 « que commencèrent les plus funestes malheurs contre la liberté des suffrages » : « On prit la liste de tous ceux qui avaient rejeté la rédaction de M. Sieyès [concernant la constitution de l'assemblée en assemblée nationale]; on fit circuler cette liste dans Paris ; tous ceux qui s'y trouvaient nommés furent représentés comme des traîtres $»^{37}$. Vérification faite dans les archives de l'assemblée, il n'existe aucune liste manuscrite

(36) Source : AN, série C 253/2, $n^{\circ} 460$.

(37) Cité dans Christine Peyrard, «Avant-propos », art. cité, p. 11. 
des 90 députés qui ont voté contre la motion Sieyès, et ce pour la raison précédemment invoquée : l'appel nominal est décompté par des bâtonnets cette fois verticaux, regroupés comme sur une règle graduée par paquet de $10^{38}$. La dénonciation des minoritaires ne peut qu'être le fait de certains députés eux-mêmes, ou des membres du public qui assistent à la séance : elle échappe totalement au contrôle de l'assemblée. C'est là une différence majeure entre la Constituante et la Convention qui parviendra, elle, à surveiller, donc à réguler, les listes d'infamie désormais « imprimée[s] par ordre de la Convention » par l'imprimerie nationale.

L'origine des listes dites « noires » qui feront florès au $\mathrm{XX}^{\mathrm{e}}$ siècle est peut-être cette liste imprimée qui paraît à l'automne 1790 sous le titre : Liste des députés plus noirs que les noires [sic] qui ont quitté la céance [sic] au moment de l'appel nominal sur la question des Ministres ; avec ceux qui ont opiné pour et contre $e^{39}$.

Sortie des presses «de l'imprimerie patriotique», cette liste est bourrée de fautes, et grossièrement imprimée. Elle recense les « noms des fuyars », les 160 députés qui ont quitté la séance pour échapper à un appel nominal rognant les prérogatives du pouvoir exécutif en signalant au roi que certains de ses ministres avaient perdu la confiance de la nation. C'est «la profonde indignation» et «la fureur» suscitées chez le rédacteur anonyme d'une « préface » vengeresse qui a motivé la rédaction de cette liste où les noms des députés ne sont pas classés, mais jetés en vrac et en pâture à une opinion publique à laquelle ils sont dénoncés comme « traîtres »: «Il est donc vrai que nous sommes trompés, trahis et lâchement abandonnés par ceux mêmes qui avaient toute notre confiance $;[\ldots] »^{40}$. Mais l'ennemi intérieur est identifié : "Presque tous ces Membres sont du Club de $89 »$ - ce qui laisse entendre que le rédacteur, lui, n'est pas de ce club modéré fréquenté par La Fayette et Mirabeau... Cette liste n'est pas vouée à demeurer unique. Elle aurait dû être suivie d'une autre liste donnant, elle, les noms des députés « qui ont opiné pour ou contre ». L'at-elle été ? Ce qui est, en revanche certain est que cette première « liste noire » de députés abstentionnistes est illégale : « Les Constituants n'ont pas voulu faire connaître à l'extérieur de l'Assemblée les résultats chiffrés des votes. La publication imprimée des résultats des scrutins a toujours

(38) Source : AN, série C 27/1, n 188.

(39) BHVP : 965797.

(40) Ibid., p. 3. 
été interdite et, en particulier, Mirabeau s'y est opposé $\rangle^{41}$. Reste que les publicistes ont régulièrement ignoré, pour ne pas dire violé, cette interdiction, ainsi que l'atteste la recension des listes imprimées. Replacées parmi les listes de dénonciation qui fleurissent à l'époque ${ }^{42}$, les listes d'infamie nées des résultats d'appels nominaux participent d'une culture de la « dénonciation civique » constitutive de l'espace public révolutionnaire. Elles rompent avec l'éthique du secret, pour laisser progressivement place à une morale républicaine de la publicité, et à un nouveau principe d'administration auxquels les députés sont soumis comme les autres fonctionnaires publics: la responsabilité. Ainsi que le dira Barère dans son intervention au procès de Louis XVI, plaidant en faveur de l'appel nominal :

«Le scrutin silencieux et secret est celui des jugemens criminels. Mais le scrutin à haute voix étant le plus solennel, et retentissant jusqu'aux extrémités les plus reculées de la république, n'en sera que plus imposant et plus pur. D'ailleurs, la nature de nos fonctions, notre responsabilité, nos mandats, tout nous force à la publicité ; c'est la garantie de la nation, c'est aussi la nôtre $»^{43}$.

\section{Représentation et responsabilité}

Un vote engage celui qui l'émet. Si le refus du mandat impératif confirme la liberté d'opiner des députés, les commettants ont le droit de connaître les opinions émises par leurs représentants non pour imposer l'exécution de leurs volontés, mais parce que, comme le précise l'article 15 de la Déclaration des droits de l'homme et du citoyen, « la société a le droit de demander compte à tout agent public de son administration ». Cet article est le socle du principe de « responsabilité » que codifiera le gouvernement révolutionnaire (décret du 14-16 frimaire an II, 4-6 décembre 1793, section V). Ainsi que le résume Michel Delon, "l'idéal du débat public suppose des intervenants - journalistes, députés, citoyens - qui s'engagent nominalement [...]. L'ancien sujet de la monarchie devient citoyen, en parlant et en votant nominalement, en assumant ses choix

(41) André Castaldo, Les Méthodes de travail de la Constituante, op. cit., p. 351.

(42) Antoine de BAECQUE, «La dénonciation publique dans la presse et le pamphlet (17891791) ", dans Harvey Chisick (ed.), The Press in the French Revolution, Oxford, The Voltaire Foundation, 1991, p. 261-279.

(43) Archives parlementaires, t. 56, séance du 4 janvier 1793, p. 202. 
individuels $\gg^{44}$. Dans cette perspective, l'appel nominal est le mode de votation révolutionnaire par excellence : «Voulez-vous déjouer beaucoup d'intrigues, et purifier les élections ? », écrit Jean-Baptiste Louvet dans son journal La Sentinelle, placardé sur les murs de Paris : «Arrêtez [que les élections] ne se feront plus dans le mystère ; arrêtez qu'on n'écrira plus; arrêtez que chacun dira hautement: Je m'appelle Un Tel et je nomme [vote] $\mathrm{Un} \mathrm{Tel}$. Voilà le scrutin digne des hommes libres $\rangle^{45}$. Surtout, l'appel nominal est appelé à devenir le mode de votation préféré des Montagnards dans la mesure où cette technique assouplit, corrige ce que l'autonomie du mandat représentatif peut avoir de rigide, sans pour autant instaurer le mandat impératif ${ }^{46}$ : dans la situation créée par l'appel nominal, « les députés ne font alors qu'un acte de sujet et non de souverain [...]. La nation reste souveraine, les avoue ou les improuve dans une seconde législature $\gg^{47}$.

Mirabeau imposait sous la Constituante l'inviolabilité parlementaire, ce droit de la personne qui assimilait la figure du député à celle du roi, sacré et irresponsable; sous la Convention, au moment de l'instauration du gouvernement révolutionnaire, en brumaire an II (octobre 1793), Barère et Billaud-Varenne devaient lui substituer un droit d'essence nouvelle : l'immunité parlementaire ou droit de la fonction. Si l'immunité parlementaire assure au député un statut particulier, lui garantit une liberté d'expression absolue au sein de l'assemblée, et interdit toute poursuite à son encontre sans l'accord préalable de l'assemblée, elle n'exclut pas que le député rende des comptes à ses commettants, lors de l'élection; à ses concitoyens, en cas de délit ${ }^{48}$. La pratique de l'appel nominal entre 1789 et 1792, qui instaure la responsabilité du vote, peut ainsi être interprétée comme une pédagogie en acte : l'appel nominal contribue à la naissance d'un droit nouveau - la responsabilité politique du député - et facilite le passage du régime de l'inviolabilité de la Constituante au régime de l'immunité parlementaire de la Convention.

(44) Michel Delon, «Le nom, la signature », dans La Carmagnole des muses, op. cit., p. 282 .

(45) J.B. Louvet, La Sentinelle, n 52, 21 août l'an 4e de l'Égalité [1792]. Journal reproduit en fac-similé, Editis, 1981 et consultable : BNF/ NUMM-9991.

(46) L'idée est de David Mongoin.

(47) Lavicomterie en 1790. Cité dans Françoise BRUNEL, "Une extrême minorité ne prouve pas le tort... », art. cité, p. 64.

(48) Anne Simonin, «Qui a tué Georges Danton? », dans Pascal Morvan (dir.), Droit, politique et littérature. Mélanges en l'honneur du professeur Yves Guchet, Bruylant, Bruxelles, 2008, p. 625-637. 
Si toute dimension tactique ou stratégique n'est évidemment pas à exclure de l'appel nominal, cette façon de voter, qui peut susciter surprise ou incompréhension de la part de l'entourage politique, comme des électeurs, est l'expression d'une intime conviction assumée. Vergniaud, député de la Gironde, n'avait-il pas déclaré à ses amis qu'il ne condamnerait jamais Louis XVI ? « Mais en arrivant au bureau, il vota la mort [...] Interpellé par ses amis sur son changement d'opinion, Vergniaud leur répondit qu'il avait cru voir la guerre civile éclater dans toute la France si Louis XVI n'était pas condamné, et qu'il n'avait pas osé mettre en balance la vie d'un individu avec le salut de la France $»^{49}$. Encore faut-il mobiliser la menace de la guerre civile pour justifier changer d'avis au moment de l'appel nominal...

La contribution de l'appel nominal à la diffusion d'une morale de la responsabilité parmi les fonctionnaires publics résulte aussi de la nature particulière d'une technique qui permet à la fois d'exprimer publiquement une opinion, et de veiller au bon exercice des fonctions de député, en remédiant à ce problème lancinant, jamais résolu des assemblées parlementaires modernes : l'absentéisme des député $\mathrm{s}^{50}$.

Avec le décret du 13 juin 1791 (tableau $n^{\circ} 1-1$ ), l'appel nominal devient une procédure disciplinaire, permettant de connaître le nom des députés présents, et de sanctionner les absents par la publicité de leur négligence. Le premier appel nominal dont les résultats firent l'objet de la première liste officielle de députés, publiée par l'imprimerie nationale, est un appel disciplinaire : la Liste des députés absens lors de l'appel nominal du 12 juillet 1791, est classée par ordre de départements, puis par ordre alphabétique, avec en regard du nom des 128 députés absents (11\%) et les «motifs de leur absence $»^{51}$. Cet appel nominal est une conséquence directe de la fuite ratée du roi par Varennes. Les députés absents, s'ils ne sont pas des traitres, sont à tout le moins des citoyens indignes de la confiance de leurs électeurs. Et la publication de leurs noms, bien davan-

(49) C.J. Mitchell, « Political Divisions... », art. cité, p. 368 et Léonard Gallois, Histoire de la Convention nationale par elle-même, op. cit., p. 335.

(50) Michel Biard, « Entre Gironde et Montagne », art. cité, p. 573

(51) Le titre de la liste figure sur la copie manuscrite qui se trouve aux AN, dans la série C 74/1/717, mais n'est pas reproduit dans la liste publiée aux Archives parlementaires, t. 28, p. 405407. Sur les controverses suscitées par les «motifs d'absence » retenus, voir l'intervention de M. Thibault, évêque du département du Cantal, président du Comité de Vérification, chargé d'établir la liste définitive, ibid., p. 213 . Voir aussi séance du 18 juillet 1791, ibid., pp. 404-407 et une rectification ibid., p. 415. La première liste officielle des députés «par ordre alphabétique des départements », hormis la Corse, est imprimée le 13 octobre 1791. Procès-Verbal de l'Assemblée nationale, Imprimerie nationale, t. I, p. 100. 
tage que la suspension un temps envisagée de leur traitement, les note d'infamie ainsi que l'atteste le débat parlementaire :

«M. Bouche : « Ma dernière proposition consiste à priver ceux qui ne répondraient pas à l'appel de leur mandat du mois de juillet. Murmures Non! Non ! [...]»

M. d'André : « [...] ce n'est donc pas par une privation d'argent qu'il faut punir ceux qui sont insensibles à l'honneur de faire leur devoir. Il vaut infiniment mieux que vous fassiez imprimer et afficher le nom des absents. Alors vous les punissez véritablement par l'endroit qui peut leur être sensible ; toute la nation saura qu'ils n'ont pas fait leur devoir, et c'est la punition la plus grave et en même temps la plus convenable que vous puissiez infliger aux représentants du peuple.

Je demande donc, M. le Président, que sans s'arrêter au moyen de supprimer le mandat qui est suivant moi un très petit moyen, et qui répugne à la délicatesse, sans s'arrêter à l'idée d'ordonner une amende, je demande dis-je, que l'Assemblée se contente d'ordonner l'impression du résultat de l'appel nominal et que ceux qui n'y auront pas été présents seront connus de la nation et leur peu de patriotisme rendu ostensible à tout le monde. Applaudissements $»^{52}$.

Hormis celui - interrompu - du 24 novembre 1791, 1'appel nominal disciplinaire ne sera renouvelé qu'à deux reprises sous les assemblées révolutionnaires, à chaque fois dans des contextes politiques extrêmement tendus : le 12 juillet 1792, après la déclaration de la patrie en danger; les 15 et 17 juin 1793, alors que 29 députés Girondins ont été expulsés de 1'assemblée quinze jours plus tôt (tableaux n 2-1 et 3).

Les sanctions qui frappent les députés «absents sans cause » sont alors considérablement renforcées. Elles font désormais l'objet d'un décret qui assimile ce type d'absence à une désertion et a pour conséquence « l'abdication volontaire » du député, sa destitution. Ces sanctions d'une sévérité extrême perdureront, sur le papier du moins, jusqu'au 20 germinal an III (9 avril 1795), date à laquelle le décret du 14 juin 1793 qui les instaure est rapporté ${ }^{33}$. Ce texte a-t-il jamais été appliqué dans toute

(52) Archives parlementaires, séance du 11 juillet 1791, t. 28, p. 114. Voir aussi l'intervention de Duquesnoy, Archives parlementaires, t. 16, 2 juillet 1790, p. 617.

(53) Les députés absents lors de l'appel nominal qui auraient dû être destitués, ne l'ont visiblement pas été. Mallarmé n'obtient pas leur remplacement systématique par leurs suppléants. Archives parlementaires, t. 68, séance du 11 juillet 1793, p. 539-540. 
sa rigueur? Nous n'avons pas d'exemple de députés destitués à cause de leur absence lors d'un appel nominal de contrôle.

Au début de la Terreur, le mode privilégié de sanction des députés absents, comme celui des autres fonctionnaires indignes, est une infamie de fait que l'on peut assimiler à une mort civique, privative non des droits civils, mais de ces droits civiques nouvellement acquis sur lequel se fonde le nouveau lien politique de concitoyenneté. Alors que le législateur avait décrété l'impression et l'insertion au Bulletin de la Convention, de la liste des noms des députés «en commission», des représentants en mission absents pour noble cause, apparaît imprimée au Bulletin de la Convention du 18 juin 1793 une nouvelle liste d'infamie : «L'état exact des députés en état d'arrestation chez eux, de ceux partis depuis leur arrestation et enfin de ceux dont l'absence n'a pas permis de mettre les mandats d'arrêt à exécution ». Les noms rendus publics sont ceux des 29 députés Girondins « suspects », « évadés » et « en fuite ». Quelques mois plus tard, le gouvernement révolutionnaire abattra une main de fer sur la représentation nationale : le 3 octobre 1793, ce sont 41 députés Girondins qui seront "prévenus de conspiration contre l'unité et l'indivisibilité de la République » et renvoyés devant le tribunal révolutionnaire par décret de la Convention.

« La Convention doit être grande, en même temps qu'elle fait acte de justice. Il faut que le décret qu'elle va prononcer soit rendu aussi solennellement que celui qui envoya le tyran à l'échafaud. Il faut que chacun se prononce dans cette circonstance, et s'arme du poignard qui doit percer le sein des traîtres. Je demande que le décret soit prononcé par appel nominal».

Billaud-Varenne, puisque c'est de lui qu'il s'agit, n'obtiendra pas gain de cause face à Robespierre qui lui répond :

« Je ne vois pas la nécessité de supposer que la Convention est divisée en deux classes, celle des amis du peuple, et l'autre des conspirateurs et des traitres. Nous ne devons pas croire qu'il y ait ici d'autres conspirateurs que ceux désignés dans le rapport. [...] Je demande que ce décret soit simplement mis aux voix $»^{54}$.

(54) Archives parlementaires, t. 75, séance du 3 octobre 1793, p. 535. 
Pourquoi Robespierre s'oppose-t-il à l'appel nominal quand il s'agit de renvoyer les députés Girondins devant le tribunal révolutionnaire ? Est-ce, comme il l'affirme, pour ne pas diviser la Convention ? Ou parce qu'il craint bien davantage un fort taux d'abstention manifestant l'indécision de l'assemblée ? Depuis 1792, l'appel nominal est l'une des techniques parlementaires favorites des Montagnards : ils en exploitent les possibilités d'obstruction en séance ${ }^{55}$ et les potentialités politiques auprès de l'opinion publique ${ }^{56}$. Et Robespierre est un trop grand parlementaire pour ignorer les pièges de l'appel nominal...

\section{La première campagne électorale ? Le Tableau comparatif des sept appels nominaux de 1792}

Si l'on en croit l'abbé Georgel, l'appel nominal est né à droite. Sous la Constituante, en effet :

« [Les factieux] s'aperçurent que l'appel nominal pour donner sa voix, occasionnait des suffrages motivés que leur dérobaient souvent les voix sur lesquelles ils avaient compté ; que d'ailleurs la nécessité de parler, la crainte de fixer l'attention, quelquefois la honte de paraître ainsi publiquement le partisan d'une motion trop répréhensible, empêchaient plusieurs de se ranger trop hautement de leur bord : ils proposèrent, en conséquence, et firent adopter le mode de voter par assis et levé. Cette forme [...] donnait à la gauche une grande supériorité ; lorsqu'elle se levait en masse pour approuver ou rejeter, elle écrasait de son poids la minorité de droite, et elle avait toujours pour elle les applaudissemens et les clameurs des galeries. [...]

Quand on prévoyait qu'il serait impossible de refuser l'appel nominal que la droite demandait très-souvent à grands cris, pour mieux connaître les vues de l'assemblée, les factieux qui en craignaient toujours l'issue,

(55) Voir la séance du 6 janvier 1793 où la Montagne s'élève contre la tentative de suppression de la permanence des sections de Paris, et où David a cette formule : «L'appel nominal ou la guerre civile ». L'appel nominal n'a toutefois pas lieu. Dans Archives parlementaires, t. 56, p. 244.

(56) C'est Saint-Just qui, le premier, le 27 décembre 1792, formula la nécessité de l'appel nominal «sur le jugement de Louis Capet» : "Je demande que chaque membre de la Convention paraisse successivement à la tribune et prononce ces mots : Louis est ou n'est pas convaincu. Après, la peine ou l'absolution sera également décrétée à l'appel nominal. [...] (Applaudissements dans les tribunes) ». Archives parlementaires, t. 55, p. 710. L'idée sera reprise et développée par Barère dans son intervention décisive du 4 janvier 1793. 
introduisaient, du côté gauche, des étrangers soudoyés, qui prenaient le nom de députés absens $[\ldots] »^{57}$.

L'appel nominal bascule à gauche sous la Législative ainsi que l'atteste le Tableau comparatif des sept appels nominaux qui ont eu lieu sur différentes questions importantes, depuis le commencement de la session, jusqu' au 10 août $1792^{58}$. Initiative des Jacobins, ce Tableau aurait été décidé suite à l'appel nominal du 8 août dont La Fayette sortit blanchi, la gauche de l'assemblée ayant échoué à faire mettre en accusation un général auquel elle reprochait un comportement factieux ${ }^{59}$ (tableau $\left.n^{\circ} 2-2\right)$. Deux mois auparavant, La Fayette avait déjà fait l'objet d'un appel nominal. Le 28 juin 1792, il était venu justifier à la barre de l'assemblée le fait d'être l'auteur d'une pétition critiquant les sociétés populaires. L'attaquant sur un problème de pure forme - le général disposait-il oui ou non d'un congé officiel ? -, la gauche échoue à le prendre en défaut. La mise en accusation de La Fayette devient dès lors une revendication relayée par les sections. Le 8 août, suite à un long débat, la gauche perd à nouveau l'appel nominal contre La Fayette. La dernière digue légale face à l'insurrection populaire vient de céder : " Quand 1'Assemblée se refusa à destituer La Fayette, le tocsin ponctuellement appela les sections aux armes » écrit Norman Hampson ${ }^{60}$.

De cet échec tactique transformé par l'insurrection populaire en victoire contre la monarchie qu'elle emporte, le girondin Louvet tire les conclusions politiques :

« L'appel nominal du [8] août me paraît avoir été [le] scrutin épuratoire [de l'assemblée]. Il me semble que rien ne peut dispenser les départemens de ré-élire ceux qui ont voté contre Lafayette dans cette séance mémorable, de même que rien ne doit engager à conserver ceux qui ont voté pour lui $»^{61}$.

De cette analyse de Louvet, la société des Jacobins de Paris va faire une véritable machine de guerre, donnant aux futurs électeurs les raisons

(57) Mémoires pour servir à l'histoire des événemens de la fin du XVIIIe siècle, depuis 1760 jusqu'en 1806-1810, par un contemporain impartial, feu M. 1'Abbé Georgel, Paris, Alexis Eymery, 1820 , t. II, p. 426-427.

(58) BHVP : 953 259. On trouve à la BNF un autre exemplaire de ce Tableau, publié en Avignon par Tournai et Sabin (BNF : 2003-86896).

(59) Patrice Gueniffey, Le Nombre et la raison, op. cit., p. 435.

(60) Norman Hampson, Danton, London, Gerald Duckworth and Co Ltd, 1978, p. 72.

(61) Jean-Baptiste Louvert, La Sentinelle, $\mathrm{n}^{\circ} 52$. 
de ne pas voter pour tel ou tel " votant-non » au décret de mise en accusation du général La Fayette. Diffusé avec une lettre du président aux sociétés affiliées en province au moment des élections à la Convention, le Tableau comparatif des sept appels nominaux va se révéler d'une grande efficacité :

«Ceux de nos représentants qui ont été fidèles au peuple vous sont désignés par l'opinion, et vous en avez la liste dans ceux qui ont voté pour le décret d'accusation contre La Fayette. Le tableau comparatif que nous vous envoyons finira de vous faire connaître et vos amis, et vos ennemis. Parmi vos constituants vous avez les Petion, les Robespierre, les Buzot, les Anthoine, les Corroller, les Grégoire, les Le Peletier, les Dubois de Crancé, le bon père Gérard, et quelques autres fidèles défenseurs des droits du peuple, en petit nombre, il est vrai, mais, sous ce rapport, plus dignes d'obtenir nos suffrages. Ceux-là on fait leurs preuves $[\ldots] \aleph^{62}$.

Et seront effectivement élus à la Convention, alors que les « votantsnon » seront impitoyablement éliminés : seulement deux d'entre-eux seront réélus ${ }^{63}$. Considéré par les électeurs de la moitié des assemblées primaires auxquels il parvint comme un document officiel ${ }^{64}$, le Tableau semble avoir provoqué une grande crainte parmi les "votants-non». Certains se crurent obligés de justifier leurs votes ${ }^{65}$; d'autres s'efforcèrent sans succès d'attirer l'attention de la Législative sur les divisions que le Tableau alimentait :

« Un membre demande que l'Assemblée nationale déclare, au nom de la patrie, qu'elle anéantit toutes espèces de listes tendantes à diviser les Citoyens, et, notamment celle qui a pour titre Tableau comparatif des sept appels nominaux, etc. L'ordre du jour invoqué sur cette demande est décrété ${ }^{66}$.

(62) «Adresse envoyée aux sociétés affiliées sur les élections prochaines », 22 août 1792, dans F. A. Aulard, La Société des Jacobins. Recueil de documents pour l'histoire du club des Jacobins de Paris, Jouhaut, Noblet, Maison Quantin, 1892, t. 4, p. 234.

(63) C.J. Mitchell, « Political Divisions... », art. cité, p. 359 et Patrice Gueniffey, Le Nombre et la raison, op. cit., p. 435 : « Parmi les 196 députés de la Législative réélus en septembre 1792, 12 seulement avaient voté avec le «côté droit » dans l'un au moins des trois appels les plus importants [...]».

(64) Alison Patrick, The Men of the First French Republic, op. cit., p. 145, p. 148. En revanche, le Tableau... fut dérobé dans l'assemblée primaire du Gard, avant que personne n'ait pu le lire! ibid., p. 173.

(65) C.J. Mitchell, « Political Divisions... », art. cité, p. 358-359.

(66) Procès verbal de l'Assemblée, Imprimerie nationale, 1792, t. XV, 8 septembre 1792, p. 119. 


\section{L'abstention ou la modération est une qualité révolutionnaire}

Le Tableau poursuivra donc sa carrière, et avec lui l'idée que l'appel nominal, instrumentalisé à des fins politiques est une technique dangereuse qui contribue à renforcer les oppositions politiques en polarisant les opinions, exprimées selon la logique binaire du oui ou du non : «Dans l'assemblée nationale, chaque député appelé répond oui ou non, et lorsque par équivoque (ce qui est arrivé quelquefois), il répond l'un et l'autre, la gaieté française ne perd point ses droits $»^{67}$. Ceux qui votent oui et non, et surtout ceux qui ne votent ni oui, ni non, n'auraient pas droit de cité dans le cadre de l'appel nominal. Cette vision de l'appel nominal est doublement inexacte.

N'est-ce pas suite à l'appel nominal demandé par le député du Gers, Jean Mouysset (1726-1810), le 23 février 1792, que « pour la première fois, à cette occasion, la presse distingua par leurs noms propres, les fractions de l'assemblée. Brissot [...] cite les feuillans, les independans, les patriotes-jacobins (son parti) et la montagne $»^{68}$ ? Cela fait, à tout le moins, plus de deux tendances politiques.

Les ni oui, ni non se rangent dans la troisième colonne de l'appel nominal qui devient la règle sous la Convention. Cette colonne dite des « absents » identifie les plus mal connus des députés, les abstentionnistes, auxquels l'appel nominal donne en quelque sorte droit de cité en les désignant négativement, nous autorisant à les qualifier ni oui, ni non. D'où un défaut majeur des tableaux présentés dans l'annexe 2 : les « taux de présence » des députés entre 1789 et 1793 , calculés à partir du cumul des oui et des non rapportés au nombre des élus, sont quasi-systématiquement sous-évalués dans la mesure où les abstentionnistes ne sont pas alors pris en compte. L'apparition des ni oui, ni non sous la Convention traduit le renforcement des contraintes qui pèsent sur l'assemblée au moment du gouvernement révolutionnaire, et la surveillance plus étroite dont sont l'objet les députés. Elle manifeste aussi à une époque de radicalisation de la Révolution, la persistance d'un type d'engagement singulier, que l'on pourrait qualifier d'extrêmement modéré.

Rassemblés dans le courant politique le plus mal connu, le centre ou la Plaine, les ni oui, ni non représentent la fraction des députés la

(67) M. DE L'ÉPITHÈTE, Dictionnaire national et anecdotique, op. cit., p. 77.

(68) Buchez et Roux, Histoire parlementaire de la Révolution française, Paulin, 1834, t. 13, p. 173 . 
moins identifiée, tant d'un point de vue politique - l'abstentionnisme est un phénomène peu ou pas étudié dans l'histoire politique française - que d'un point de vue biographique - Mitchell notait en 1988 l'attention accordée aux votants-oui, quand deux votants-non seulement avaient fait l'objet d'une biographie. Reste qu'il y a plus inconnu encore que les votants-non, les votants ni oui, ni non ou abstentionnistes.

$\mathrm{Si}$, en sus des ni oui ni non, l'on tient compte de ceux qui votent oui mais, ou non mais, comme lors de l'appel nominal concernant Carrier (annexe 3), surgit une population, certes minoritaire on va le voir, qui en refusant (cas des abstentionnistes) ou en subvertissant les catégories autorisées (cas des oui mais, non mais) affirme par son vote inclassable que, contrairement à la fameuse loi de Solon, il est possible, même dans des contextes de tensions politiques extrêmes, de rejoindre un camp politique sous condition, voire de revendiquer le fait de ne pas prendre parti ${ }^{69}$. Comment caractériser cette position extrêmement modérée ou modérée révolutionnaire, modérée dans la mesure où elle s'exprime par le ni oui, ni non; révolutionnaire, dans la mesure où elle s'assume dans le cadre de l'appel nominal?

L'abstention est un phénomène massif pendant la Révolution. Si le phénomène de l'abstention électorale est connu, et a fait l'objet d'une socio-histoire avant l'heure ${ }^{70}$, le sens et le contenu politique de l'abstentionnisme parlementaire en l'an II n'a, à notre connaissance, jamais été étudié. Les appels nominaux, qui permettent de déployer le nuancier de l'abstention, ce refus volontaire d'exercice du droit de vote sur des questions présentées comme fondamentales pour la survie de la communauté politique, pourraient aussi se révéler décisif pour comprendre la radicalité du non choix formulé par les députés qui se refusent à voter oui ou non.

L'abstention est, lors de l'appel nominal, un acte militant, perçu comme tel par les contemporains : «Les membres de l'Assemblée qui ne sont pas compris dans cet appel [celui du 4 mai 1791 concernant le rattachement d'Avignon et du Comtat-Venaissin à la France], sont absents ou malades : plusieurs sont sortis au moment de l'appel, entr'autres : Messieurs La Fayette, Bailly maire de Paris, d'André, l'abbé Sieyès » : aux

(69) Solon, au début du $\mathrm{V}^{\mathrm{e}}$ siècle avant J.C. aurait introduit à Athènes la loi suivante rapportée par Aristote : «[...] Celui qui dans une guerre civile ne prendra pas les armes avec un des partis sera frappé d'atimie et n'aura aucun droit politique », dans Aristote, Constitution d'Athènes, Les Belles Lettres, 1996, p. 19.

(70) Patrice Gueniffey, Le Nombre et la raison, op. cit., p. 192-232 : «L'abstention, une interprétation sociologique » et Malcolm CROOK, Élections in the French Revolution, Cambridge UK, Cambridge University Press, 1996, p. 92-95, 98-100. 
côtés des absents, des malades, des «fuyars », les secrétaires inventent une nouvelle catégorie, les «point de voix » qui eux, ne quittent pas l'assemblée, mais refusent publiquement d'opiner par oui ou par non ${ }^{71}$.

Que recouvre cette expression, point de voix ? On sait seulement par la liste imprimée que M. Cheynet est classé « point de voix » car il «a déclaré qu'il s'abstenoit de voter parce qu'il était possesseur d'une partie du pais ». Quid des 45 autres députés qui l'accompagnent? On l'ignore.

C'est sous la Convention que le législateur va s'efforcer de serrer au plus près la réalité de l'abstention, en multipliant les catégories de répartition de ceux qui ne votent pas. C'est très net, par exemple, lors du premier appel nominal concernant la culpabilité de Louis XVI (15 janvier 1793) ou, à côté des oui mais (32) sont recensés ceux qui « ont fait diverses déclarations sans répondre à la question posée » (3), ceux qui «se sont récusés ou abstenus » (10), soit 13 abstentionnistes ${ }^{72}$. Concernant la mise en accusation de Marat (13-14 avril 1793) : 47 députés se retrouvent dans la catégorie « point de vœu quant à présent », 7 demandent l'ajournement, 3 se récusent, 1 motive son vote « en termes équivoques », soit 58 abstentionnistes.

Ce qui apparaît dans les "grands » appels nominaux, se vérifie aussi dans les appels nominaux « importants », tel celui du 28 mai 1793 concernant la suppression de la Commission des Douze, mise sur pied par les Girondins pour surveiller la commune de Paris. On l'a vu, sur la liste manuscrite apparaissent les noms de 12 députés ayant voté "blanc» (illustration $\left.\mathrm{n}^{\circ} 1-\mathrm{C}\right)$. C'est peut-être en étudiant précisément cette population de députés, en s'attachant en particulier au fait de savoir si l'abstention est pour eux un choix unique ou est une pratique régulière que l'on parviendrait à comprendre les enjeux politiques du ni oui ni non: les ni oui, ni non viseraient à maintenir, à stabiliser l'ordre public révolutionnaire, quand les votants-non s'efforceraient de faire rétrograder, et les votants-oui de révolutionner la Révolution?

De Pierre ou Jean-Pierre Malhes, ou Mailhe (1748-1829), on sait seulement qu'il fut élu député suppléant du Cantal, et qu'il siégea à la Convention entre le 17 janvier et le 7 octobre 1793. En réalité, un temps beaucoup plus bref puisque selon A. Kuscinski, le 3 juin 1793, Malhes fut nommé représentant en mission en Lozère, et ne reparut plus ensuite à

(71) BNF : LE-29-1904 et Michel BiARD, «Entre Gironde et Montagne », art. cité, p. 567.

(72) Archives parlementaires, t. 57, p. 99. 
l'assemblée... Ses six mois d'exercice de la fonction de député se signalent par un abstentionnisme forcené. Fils de marchand, président du tribunal de commerce d'Aurillac, Malhes qui remplace son frère Joseph, défaillant, ne se précipite pas à la Convention. Il ne vote « ni verbalement, ni par écrit ", lors des trois premiers appels nominaux concernant Louis XVI. Et quand il « prend séance », le 17 janvier, il refuse « de voter sur la question du sursis » : «Le Moniteur est ici plus exact que le procès-verbal qui le porte absent par maladie. Le 13 avril, il refusa encore de se prononcer sur la question de la mise en accusation de Marat $»^{73}$.

L'abstentionnisme n'est pas chez Malhes le signe extérieur d'une opinion révolutionnaire tiède. On signale qu'en 1789 il «adopta avec enthousiasme les idées de la Révolution », que le 4 août 1791, «il s'engagea à entretenir deux gardes nationaux », ce pour quoi la Constituante lui accorda 《 une mention honorable $»^{74}$. Son enthousiasme révolutionnaire ne semble pas avoir fléchi avec le temps. Dans sa lettre de démission, il renouvelle le «serment que je fais de vivre et de mourir républicain $[\ldots]$ et de propager dans mon pays, autant qu'il sera en moi, les principes de la liberté et du républicanisme ». L'on ne doit pas tirer de ce document lu à la tribune de l'assemblée le 6 octobre 1793 de conclusions trop hâtives : ce républicanisme enflammé n'est-il pas de pure tactique ? Reste qu'à quatre jours de l'institution du gouvernement révolutionnaire, et de la mise en place de l'état de siège politique dans lequel toutes les forces de la nation sont mobilisées plus connu sous le nom de Terreur, la Convention laisse Malhes se retirer pour raisons personnelles ${ }^{75}$ : le fait est en soi exceptionnel, et prouve à tout le moins que si Malhes n'a pas servi avec zèle, il n'a pas desservi la patrie, et que son attitude politique n'a suscité aucun soupçon. Est-ce parce que lors de l'appel nominal du 28 mai 1793, concernant la suppression de la Commission des Douze exigée par la Montagne, Malhes a abandonné le camp des abstentionnistes, et lui a accordé son soutien en votant non?

L'appel nominal n'est pas l'ennemi du centre. Il permet au contraire aux députés constituant le noyau dur de la Plaine, d'exprimer leur identité, leur modération extrême, tout en n'excluant pas la conclusion d'alliances qui, les transformant en votant-oui ou votant-non selon les circonstances en font des alliés temporaires soit de la droite, soit de la gauche de l'assemblée.

(73) A. Kuscinski, Dictionnaire des Conventionnels, F. Rieder, 1916, p. 230.

(74) A. Robert et G. Cougny, Dictionnaire des parlementaires français. Consultable sur le site internet de l'Assemblée nationale.

(75) Je remercie Serge ABERDAm pour ce commentaire. 
Ce qui distinguerait ainsi l'abstention, des comportements politiques d'adhésion exprimés par oui ou non, serait non pas une radicalité moindre, mais une plus faible cohérence. Reste que le votant ni oui, ni non manifeste l'existence trop souvent inaperçue d'un droit à la dissidence au sein de la communauté politique, au moins dans la première moitié de l'an II. Ce droit est certes exceptionnel (rareté relative des appels nominaux), et fragile (complète disparition des appels nominaux après juin 1793). Les choses eussent-elles été différentes si la technique de l'appel nominal avait été codifiée, et si l'appel nominal était apparu non plus comme une manière de voter subsidiaire, mais comme le mode institutionnel d'expression de la minorité ?

L'appel nominal, on l'a dit, a fait l'objet de deux essais de codification. Le premier revêt la forme d'une machine destinée à simplifier le décompte des voix. Présentée comme « anecdotique » par André Castaldo, cette machine est, en réalité, d'une grande modernité : les machines à voter ne sont-elles pas aujourd'hui en vigueur dans tous les parlements ? $^{76}$ L'autre essai de codification est plus politique : il vise à la fois à inscrire une nouvelle disposition dans la Constitution, et à modifier le règlement de la Convention. Ces tentatives de codification, matérielle et constitutionnelle, de l'appel nominal échoueront. Il est toutefois essentiel de rappeler leur existence non tant pour établir les responsabilités historiques dans la violence extrême subie par la représentation nationale en l'an II que pour insister sur le fait que le gouvernement d'exception connu sous le nom de gouvernement révolutionnaire, contenait aussi en germe la possibilité d'une « république démocratique $»^{77}$.

\section{L'échec de la codification matérielle : la machine à appel nominal de François-Élie Guirault}

Censé remédier à un vote douteux par assis et levé, l'appel nominal se révèle être une source d'erreurs sans nombre, ainsi que l'attestent les demandes en rectification des votes que les députés font paraître dans la

(76) André Castaldo, Les Méthodes de travail de la Constituante, op. cit., p. 353, note 202. Sur les rapports entre technique et démocratie consulter Delphine Gardey, Écrire, calculer, classer : comment une révolution de papier a transformé les sociétés contemporaines (1800-1940), Éditions La Découverte, 2008, p. 25-48 pour la période révolutionnaire.

(77) Françoise Brunel, «Introduction », dans Billaud-Varenne, Principes régénérateurs du système social, Publications de la Sorbonne, 1992, p. 47-50. 
presse. Les défauts de ce mode de recueil des voix n'échappent pas au citoyen Guirault qui s'efforce, très tôt, d'y remédier.

De François-Élie Guirault, né en 1760 à Bordeaux, de taille « d'environ cinq pieds six pouces, âgé de quarante ans, cheveux bruns, extrêmement maigre, les yeux forts cavés, la peau bileuse et tachée de rouge », on sait qu'il renonça à l'état de notaire, et fit fortune dans le commerce ${ }^{78}$. Monté à Paris en 1785, il s'y trouve au moment de la Révolution. «L'homme par excellence » de la section du Contrat Social qu'il présidera, membre du club des Jacobins, Guirault fait partie, selon DécembreAlonnier, de ce « grand nombre d'individus [...] voulant à tout prix jouer un rôle politique », qui se distinguent en pétitionnant à la tribune. Passionné par le phénomène parlementaire, Guirault multiplie les inventions destinées à améliorer le fonctionnement de l'assemblée et la transmission de la parole parlementaire au public. Resté dans l'histoire comme inventeur du logotachigraphe, procédé "par lequel il écrit aussi vite que la parole $»^{79}$ en vue de restituer l'exactitude des débats qu'il publie dans le Le Logographe, journal national, Guirault semble être le seul à s'être intéressé à l'appel nominal ainsi que l'atteste le Tableau d'appel nominal présenté par lui à la barre de la Constituante, le 30 avril 1791.

De ce Tableau d'appel nominal dont Guirault a dû faire un dessin, et a, de façon certaine, réalisé une maquette, nous n'avons plus traces que par les descriptions reprises par les différents journaux ${ }^{80}$. Reste que la description la plus précise est ailleurs, jointe aux documents relatifs à la séance de la Constituante où fut présenté le Tableau décrit aux commissaires de l'Assemblée en ces termes :

(78) Guiraut au calomniateur Chery, membre du comité de surveillance, et à ses co-dénonciateurs, Balardelle, Paly, Robert, Gentil et Potet, tous de la Section du Contrat social, 15 jour du $2^{\mathrm{e}}$ mois l'an II de la République, une et indivisible, p. 2. Voir la notice « Guirault, François-Élie» dans Albert Soboul, Raymonde MonNiER (dir.), Répertoire du personnel sectionnaire parisien en l'an II, Paris, Publications de la Sorbonne, 1985, p. 145.

(79) Procès-verbal de la séance du 23 mars 1792, rapport de Romme, dans James GullLaume, Procès Verbaux du Comité d'Instruction de l'Assemblée Législative, Paris, Imprimerie Nationale, 1889 , p. 156.

(80) Le Moniteur, t. XXV, 30 avril 1791, p. 472-473. 
« Messieurs,

Depuis que l'Assemblée national est occupée de ses pénibles fonctions, je me suis voué d'une manière particulière à l'utilité de ses travaux.

Le 21 juillet dernier [1790], je lui offris, et l'assemblée nationale a bien voulu l'agréer, une gravure qui présente l'art d'écrire mot à mot et en toutes lettres aussi vite que la parole : plan que j'avais déjà soumis il y a dix-huit mois au comité des Règlements.

L'appel nominal m'ayant paru mériter qu'on s'occupat d'un moyen facile et prompt pour économiser du tems à l'assemblée ; délivrer messieurs les secrétaires de cette attention fatiguante qui craint les distractions ; anéantir jusques à l'ombre des doutes s'il pouvoit y en avoir; enfin rendre le public témoin de la justice et de l'évidence de l'appel en évitant la plus petite erreur.

Quelques instants de recherche et d'application m'ont fait trouver, Messieurs, le moyen désiré que j'ai raproché le plus possible de cet état de clarté et de simplicité.

J'ai imaginé une certaine quantité de tubes de verre réunis ensemble sure une même ligne ; je les ai divisés en trois masses de manière qu'elles puissent offrir sur le même plan, un tableau d'appel positif, un tableau d'appel négatif, un tableau d'abstention. J'ai encadré ces masses une à une. Je les ai ornées d'un ordre corinthien.

Ces tubes placés dans le sens vertical des colonnes, leur base repose sur un réservoir commun à chaque masse ; leur sommet passe au travers d'un entablement qui sert de portique ; leur embouchure reçoit un entonoir; des boules d'un égal diamètre et aux trois couleurs nationales sont jetées dans ces tubes selon l'appel, positif, négatif ou absent ; des parallèles tirées divisent ces boules de cinq en cinq; l'addition des nombres est faite dans le haut et à mesure que l'entonoir change de tube l'addition multipliée se trouve ainsi faite, de manière que l'appel nominal fini, tout le monde, après en avoir été le sévère juge, peut dire aussitôt quel est le résultat au vrai des suffrages.

J'ai cru devoir vous soumêttre, messieurs, cette invention nouvelle qui présente une utilité réelle. Lorsque vous l'aurez examinée, vous vous convaincrai de sa simplicité, et de ses agréables effets. Je désire, Messieurs, qu'elle reçoive votre approbation ; les citoyens, vous le savez, ont besoin d'encouragement; les représentants de la nation qui savent juger du vrai mérite des choses savent aussi être justes.

Veuillez me faciliter, je vous prie messieurs, les moyens de faire hommage de mon travail à l'Assemblée Nationale. Je me chargerai de l'exécution en grand pour son utilité. J'en ferai soigner les imperfections. Je désire Messieurs le faire à mes frais d'après ce que vous en aurez décidé 
et je m'en acquiterai d'une manière aussi digne de la majorité de l'Assemblée Nationale que de mon patriotisme, et de mon dévouement à la chose publique $[\ldots] \gg{ }^{81}$.

Il y aurait beaucoup à dire sur cette lettre, qui relève à la fois du prospectus publicitaire et de la supplique. Renvoyé au Comité de Constitution, le Tableau d'appel nominal fait l'objet d'un rapport favorable de Le Chapelier, soulignant la fiabilité et la célérité du procédé : «Trois tableaux, ayant chacun deux scrutateurs pour le servir, suffisent pour une assemblée de 600 personnes, et un tour de scrutin se fait en moins de trois quart d'heure $\gg{ }^{82}$. La longueur de l'appel nominal est l'un des principaux obstacles à sa mise en œuvre - «N'est-il pas constant qu'il faut cinq heures à chaque appel nominal $[\ldots]$ ? $\gg^{83}$. Avec la machine imaginée par Guirault, ce handicap disparaît.

L'appel nominal tel que l'envisage le jacobin Guirault ne se déploie pas selon une logique binaire, il ne distribue pas les députés entre les camps irréductibles des votants oui et des votants non, mais tient compte des abstentionnistes : "J'ai imaginé une certaine quantité de tubes de verre réunis ensemble sure une même ligne; je les ai divisés en trois masses de manière qu'elles puissent offrir sur le même plan, un tableau d'appel positif, un tableau d'appel négatif, un tableau d'abstention ». De couleur probablement blanches, les boules du «tableau d'abstention » participent avec les boules rouges du «tableau d'appel positif », et les bleues du « tableau d'appel négatif » à l'expression légitime de l'opinion parlementaire, officialisant en quelque sorte la possibilité de ne pas prendre parti, acculturant auprès des députés le fait que le refus de prendre parti franchement assumé lors d'un scrutin public est une stratégie révolutionnaire digne de ce nom.

Au vu de l'enthousiasme de Guillotin alors commissaire de la Salle, face à l'intérêt de « la machine inventée par le sieur Guirault, [qui] en mettant le public, comme l'Assemblée nationale, dans la possibilité de voir d'un seul coup d'œil le recensement des voix, donne toujours un résultat précis et certain; la simplicité et l'utilité de cette machine sont évidentes ${ }^{84}{ }^{8}$, on se prend à concevoir pour le Tableau d'appel nominal un

(81) AN, série C 126, dossier 420.

(82) Séance du 15 juin 1791, Archives parlementaires, t. 27, p. 237.

(83) Opinion de Féraud, séance du 23 thermidor an II (10 août 1794), Archives parlementaires, t. 94, p. 32.

(84) Archives parlementaires, t. 25, séance du 30 avril 1791, p. 473. 
succès comparable à celui rencontré par une autre machine, « d'une simplicité » et d'une « utilité » tout aussi évidente : la guillotine. Ce ne sera pas le cas. Ardemment soutenu par Le Chapelier ; violemment combattu par Gaultier-Biauzat, le Tableau d'appel nominal aurait dû être utilisé par l'Assemblée pour «l'élection prochaine de son nouveau président ${ }^{85}$. Cela ne semble pas avoir été le cas ${ }^{86}$.

Le Tableau d'appel nominal ne tomba toutefois pas dans l'oubli : le 28 mars 1792, le Comité d'Instruction publique confiait au citoyen Gentil (1755-1830), avocat, député du Mont-Blanc, « l'examen du logoscope, présenté à l'Assemblée nationale par M. Guirault », lui demandant « de rendre compte en même temps d'un nouveau mode d'appel nominal du même auteur $\rangle^{87}$. François Gentil a-t-il jamais rédigé le rapport demandé ? Les ennuis politiques de Guirault, démissionnaire de la présidence de sa section le 29 juillet 1793, arrêté et détenu comme suspect, échappant à la guillotine, le 9 thermidor, devaient être peu propices au succès de ses différents projets en l'an II...

\section{L'impossible codification constitutionnelle : la minorité a-t-elle des droits ?}

« De quoi s'agit-il au fond? Un homme [le citoyen Leroux, juge de paix de la section de l'Unité] prévenu d'avoir tenu les propos les plus inciviques $[\ldots]$ cet homme a été arrêté par ordre d'une autorité constituée [le comité révolutionnaire] [...] quelques individus se présentent à la barre pour réclamer ce citoyen; qu'avez-vous fait? Sans entendre la municipalité $[\ldots]$ sans même oser prononcer sur le fond, puisque vous avez renvoyé au comité de législation, vous rendez provisoirement la liberté à cet individu; vous allez donc aussi la rendre à tous les contrerévolutionnaires arrêtés dans les départements ? $\|^{88}$

C'est en ces termes que le jacobin Couthon résume la question qui, ce 17 mai 1793, enflamme la Convention, mettant aux prises d'un côté les Montagnards, qui exigent le recours à l'appel nominal, sur une question

(85) Intervention de Merlin, séance du 15 juin 1791 citée.

(86) Alexandre de Beauharnais succède à M. Dauchy comme président de l'Assemblée, le 18 juin 1793. Les Archives parlementaires ne donnent aucune précision sur le mode de scrutin, simplement son résultat (t. 27, p. 319-320). Rien non plus dans la série C : AN, C 257/1/503.

(87) James Gulllaume, Procès-verbaux du Comité d'Instruction de l'Assemblée Législative, op. cit., p. 160.

(88) Le Moniteur, t. 16, séance du 17 mai 1793, p. 411-412. 
qui eût pu être la suivante : «Le citoyen Leroux doit-il être maintenu en état d'arrestation jusqu'à ce que le comité de Législation ait remis son rapport? Oui ou non. »; de l'autre, la Gironde, et la Plaine, - la Gironde surtout puisque ce sont les orateurs les plus en vue de ce courant, Vergniaud et Guadet, qui s'affrontent avec les Montagnards, Couthon et Legendre au premier plan, Danton, - et la précision a son importance -, est à la manœuvre.

Malgré sa mobilisation, - 150 Montagnards signent la demande d'appel nominal déposée sur le bureau du président ${ }^{89}$-, la gauche de l'assemblée n'obtient pas gain de cause : l'appel nominal n'a pas lieu. Mais les échanges auquel donne lieu son invocation sont importants dans la mesure où ils soulèvent et apportent, au moment de la discussion et de l'adoption de la nouvelle Constitution, une réponse originale à deux questions de principe : celle du droit de résistance et celle du droit de la minorité.

Reconnu par la Déclaration des droits de l'homme et du citoyen de 1789 (art. 2), le droit de résistance sort renforcé de la Déclaration de 1793 qui en fait, en certaines circonstances, « le plus sacré des droits et le plus indispensable des devoirs » (art. 35). Ce droit est au cœur du débat de la séance du 17 mai 1793. La majorité, menée par les Girondins, est d'avis qu'il convient de mettre en liberté le citoyen Leroux. La minorité, constituée par la Montagne, est contre. Les effectifs de la Montagne (40,3\%) sont normalement supérieurs à ceux des Girondins $(23,8 \%)^{90}$. Ces derniers se trouvent toutefois favorisés par la conjonction de deux faits en ce mois de mai 1793 : «Et le départ de l'armée parisienne, que Santerre avait accompagnée, et le départ des commissaires de la Convention, presque tous pris parmi les membres qui siégeaient à la Montagne $»^{91}$. De cette infériorité numérique temporaire, les Montagnards tirent une théorie républicaine de la minorité, ainsi formulée par le dantoniste Legendre : « La majorité ne peut faire la loi à la minorité, lorsque celle-ci a pour elle les principes. [...] il y a ici oppression, nous avons le droit d'y résister [...]. C'est la minorité qui sauva la France le 10 août et le 2 septembre ; c'est la minorité qui la sauvera encore. Un membre : Faites taire ce boucher ! $[\ldots] »^{92}$ Couthon fait écho :

(89) Cette liste ne figure pas dans la série C 253/449 et 456 qui rassemblent les éléments ayant trait à la séance des 17 et 18 mai. C'est très dommage parce que ce document eût établi de façon irréfutable la composition de la Montagne.

(90) Voir note 9.

(91) Léonard Gallois, Histoire de la Convention nationale par elle-même, Chez Auguste Mic, 1834, t. II, p. 364.

(92) Archives parlementaires, t. 65, séance du 17 mai 1793, p. 15-16 ; p. 17. 
« Je suppose qu'une majorité perverse... propose et fasse décréter des mesures désastreuses, le retour même de la tyrannie, eh bien ! oserez-vous dire, dans ce cas, que votre règlement ne permettrait pas à la minorité, restée fidèle aux principes et à ses serments, de demander l'appel nominal et de résister à l'oppression ? (Rires ironiques à droite) ${ }^{93}$.

Dans un article important sur le droit de résistance, Alessandro Fontana oppose le projet de constitution girondin présenté par Condorcet le 23 février 1793, qui « invoquant la résistance comme moyen légal s'efforce de l'inscrire dans des formes juridiques strictes où les faits d'oppression sont nettement nommés et qualifiés », au projet montagnard de Robespierre, présenté le 24 avril 1793, qui « sur fond de guerre civile [...] reformule la résistance non plus comme un droit, mais comme un devoir et une voie de fait, dans une forme délégalisée ${ }^{94}$. Il est frappant de noter que le 17 mai 1793 tend un miroir inversé à cette opposition entre Girondins et Montagnards : c'est la Montagne qui se bat pour que l'appel nominal, qui institutionnalise le droit de résistance de la minorité, soit codifié, et le règlement de la Convention modifié. Couthon met en forme une proposition de Danton, et exige : «Que toutes les fois que 100 membres demanderont l'appel nominal dans des questions constitutionnelles et de législation cet appel nominal soit accordé $\rangle^{95}$. Le girondin Vergniaud prend alors la parole, plaide en faveur de la mise en liberté du citoyen Leroux, mais convient avec Couthon « qu'il est des occasions où la minorité peut croire important pour sa gloire de constater quelle a été l'émission de son vœu, et où il serait tyrannique de le lui refuser $\gg{ }^{96}$. La proposition de Couthon est renvoyée au Comité de Législation, et fait l'objet le lendemain, 18 mai, d'un projet de décret présenté par Lanjuinais qui contient les « articles additionnels au règlement pour déterminer les cas où une partie de l'Assemblée aura le droit de demander un appel nominal » :

«Art. $1^{\text {er }}$ : L'appel nominal sera fait sur les questions constitutionnelles, toutes les fois qu'il sera demandé par cent membres de la Convention, qui auront souscrit à cette [demande] sur le bureau des secrétaires.

(93) Ibid., p. 21.

(94) Alessandro FontanA, « Du droit de résistance au devoir d'insurrection », dans JeanClaude Zancarini (dir.), Le Droit de résistance XIIe-XXe siècle, Paris, ENS Éditions, 1999, p. 21-22.

(95) Archives parlementaires, t. 65, séance du 17 mai 1793, p. 21.

(96) Ibid., p. 22. 
Art. 2 : Il sera fait sur les autres questions, toutes les fois qu'il sera réclamé par cent cinquante membres, qui auront de même souscrit leur demande.

Art. 3 : En cas d'appel nominal, tout membre a la faculté de motiver sommairement son opinion ${ }^{97} \gg$ [souligné par nous]

C'est alors que Guadet intervient avec une rare violence. Il dénonce dans ce projet le moyen pour la Montagne, en légalisant « la scandaleuse résistance de la minorité », de «mettre la minorité en état de révolte habituelle contre la majorité $\gg{ }^{98}$. L'argument massue pour s'opposer à l'adoption du décret est la référence à un épisode de l'histoire anglaise, connu « sous le nom de la purgation du Parlement ». " Cet attentat dont Pride, poursuit Guadet, de boucher devenu colonel, fut l'auteur et le chef : 150 membres furent chassés du Parlement, et la minorité, composée de 50 ou 60 membres, resta maîtresse du gouvernement. Savez-vous citoyens ce qu'il arriva? Ces patriotes par excellence, instruments de Cromwell, auxquels il fit faire folie sur folies furent chassés à leur tour. Leurs propres crimes servirent de prétexte à l'usurpateur $\gg{ }^{99}$.

Le parallélisme historique est limpide: Legendre n'est-il pas comme Pride « boucher » de son état? Si la Convention codifie l'appel nominal, elle pave la voie, sous prétexte de respect des droits de la minorité, et de légalisation du droit de résistance, à l'anarchie et à un futur Cromwell, perspective qui fait autant frémir la Gironde, que certains théoriciens de la Montagne, tel Billaud-Varenne voyant dans le gouvernement militaire, ou «stratrocratie » cromwellienne, le pire des régimes politiques (après la théocratie). Le projet de décret de Lanjuinais, partiellement adopté, sera renvoyé, sur proposition de Cambacérès, au Comité de Législation: l'appel nominal ne sera de droit que sur les questions constitutionnelles (tableau $n^{\circ} 1-3$ ). C'est bien davantage la Constitution de l'an I qui conservera trace de la volonté montagnarde d'un élargissement beaucoup plus vaste du champ d'application de l'appel nominal : «Cinquante membres ont le droit d'exiger l'appel nominal » précisera l'article 50.

L'opposition des Girondins à l'institutionnalisation des droits de la minorité par le biais d'une demande d'appel nominal sur n'importe quel sujet le 18 mai 1793, les laissera fort dépourvus au moment du coup de

(97) « Projet sur les appels nominaux », texte imprimé. BHVP : 964647.

(98) Archives parlementaires, t. 65, séance du 18 mai 1793, p. 39.

(99) Ibid., p. 38-39. 
force du 2 juin et de l'arrestation de 29 d'entre eux. Les dispositions de la Constitution de l'an I ne sont alors pas en vigueur, et, à cause de leur opposition quinze jours plus tôt, l'appel nominal qui ne peut être mobilisé que sur les questions constitutionnelles, n'est toujours qu'une technique subsidiaire de vote...

«Le décret [d'arrestation] est à peine prononcé qu'un grand nombre de députés sont venus au bureau réclamer contre, et ont signé diverses déclarations pour qu'il constate qu'ils n'approuvent point ce décret et qu'ils n'ont point pris part à la délibération ${ }^{100}$. « N'a pas pris part au vote » est, on l'a vu, une des catégories de l'appel nominal. Si ce dernier avait été mis en œuvre le 2 juin 1793, les Girondins eussent-ils été exclus si facilement de la Convention?

L'appel nominal est autre chose qu'une technique de vote écrivionsnous au début de cet article. Entre 1792 et 1793, l'appel nominal met en œuvre une théorie originale de la représentation, la « représentation responsable ». Ni blanc-seing, ni mandat impératif, la représentation responsable noue un type nouveau de rapport entre les députés et leurs commettants, un rapport fondé sur la reddition de compte, les députés faisant connaitre des opinions que les commettants peuvent censurer en ne les réélisant pas, ce qui sera, on l'a vu, le cas lors de l'élection de la Convention en septembre 1792 : la diffusion du Tableau comparatif des sept appels nominaux joua alors un rôle fondamental dans le renouvellement du personnel parlementaire en provenance de la Législative.

L'étude de la technique de l'appel nominal recèle une autre histoire politique des années 1789-1795, une histoire moins obsédée par l'identification de l'ennemi, et la distribution des députés en deux camps, les votants-oui et les votants-non, que travaillée par l'expression et l'identification d'un centre, les votants ni oui, ni non, ces abstentionnistes auxquels l'appel nominal donne un nom et la possibilité d'afficher une opinion réfractaire.

L'appel nominal participe de l'écriture d'une histoire de la Montagne où le droit joue un rôle plus important que généralement admis. Codifier l'appel nominal revenait, on l'a vu, à légaliser le droit de résistance. Dissociant le droit de résistance de l'insurrection pure et simple, l'appel nominal, étendu aux questions fondamentales de l'ordre public révolutionnaire (constitution comprise), apparaissait comme un droit de 
la minorité, qui se trouvait ispo facto institutionnalisée sans que soit mise en péril l'expression de la volonté générale. Le principe majoritaire sortait, en effet, renforcé de l'épreuve de l'appel nominal. Or, comme le disait Barère, «la majorité seule a le droit de vouloir et d'exprimer la volonté générale, en état social, en législation et en jugement $»^{101}$.

Technique favorite de vote des Montagnards à partir de 1792, l'appel nominal était en particulier devenu l'une des armes de prédilection du dantoniste Legendre pour faire obstruction dans les débats. Sauf, étrangement, le 11 germinal an II (31 mars 1794), jour où Danton et les députés dantonistes furent renvoyés devant le tribunal révolutionnaire. Le fait est célèbre : seul Legendre osa monter à la tribune et prendre la parole en faveur du leader des Indulgents. Mais pourquoi ne demanda-t-il pas que le décret de renvoi devant le tribunal révolutionnaire de Danton et de ses co-inculpés fut adopté par appel nominal, alors que l'idée de l'appel nominal rôdait dans la Convention - « Nous étions une poignée d'hommes résolus à demander l'appel nominal pour connaître les désorganisateurs du gouvernement $»^{102}$ ? Il n'existe aucune explication satisfaisante à cette omission qui devait se révéler une erreur stratégique majeure... Enfin si l'on en croit Couthon.

Lors de la séance des Jacobins, du 26 germinal an II (15 avril 1794), dix jours après l'exécution de Danton et de ses co-inculpés, François Gentil (celui-là même qui fut chargé du rapport sur le Tableau d'appel nominal de Guirault, voir supra) se heurte à Robespierre. Pour emporter l'adhésion quant à l'exclusion de Gentil de la société des Jacobins,

«Couthon instruit la Société que plusieurs citoyens lui ont rapporté que, le jour de l'arrestation de Danton, Delacroix et autres, Gentil courait dans la Convention avec deux pistolets, engageant les membres à faire des motions contre le Comité de salut public. [...]»

Cette activité séditieuse de Gentil ne se limite pas au port ostensible de « deux pistolets [...] achetés le matin même dans la vente de PhilippeÉgalité », député en état d'arrestation ${ }^{103}$.

« Un membre confirme la déclaration de Couthon; il annonce qu'il vit Gentil courir dans la salle de la Convention, le jour de l'arrestation de Danton et Delacroix, cherchant à exciter les esprits et demandant qu'il y

(101) Ibid., t. 56, séance du 4 janvier 1793, p. 214, note.

(102) Journal Universel, $\mathrm{n}^{\circ}$ 1589, 12 germinal an II, p. 7293.

(103) A. Kuscinski, Dictionnaire des Conventionnels, op. cit., p. 291. 
eût un appel nominal, pour connaître, disait-il, les bons et les mauvais députés ».

François Gentil est exclu le même jour de la Société des Jacobins. Décrété suspect, son dossier ne contient qu'une feuille, un certificat médical en date du 10 germinal an II (30 mars 1794) : « Nous lui avons conseillé de profiter de la belle saison pour se rendre dans son département où il pourra user des [eaux minérales chaudes en bains et en douches] seuls [moyens] qui puissent contribuer à son rétablissement $»^{104}$. Après la séance des Jacobins, le conseil était bon.

L'appel nominal ne s'est jamais remis de la mort de Danton. L'affirmation peut paraître paradoxale, sinon infondée. Lorsque les 6-8 brumaire an III (27-29 octobre 1794), Merlin de Douai repensera l'immunité parlementaire, multipliant les garanties pour les députés, il sera précisé "[qu']il ne pourra être rendu de décret d'accusation qu'à l'appel nominal $»^{105}$. Mais ne nous y trompons pas : ce «code de brumaire», bouclier juridique pour une profession durement éprouvée pendant la Terreur, ne sera, en réalité, appliqué qu'une seule fois, lors de la mise en accusation de Carrier ${ }^{106}$. Sous la Convention thermidorienne, frappé de l'opprobre et des dérives intimidatrices de l'élection à haute voix, l'appel nominal n'a plus cours que pour des questions techniques (renouvellement du bureau de l'assemblée) et encore devient-il secret à partir du 5 thermidor an III (23 juillet 1795) (tableau $\mathrm{n}^{\circ} 1-3$ ).

Anne SimOnIN

CNRS, IRICE, Université de Paris I et de Paris IV 8 rue du Fbg Poissonnière, 75010 Paris simonin.anne@wanadoo.fr

Corinne Lechevanton-Gomez IHRF - Université de Paris I - Panthéon-Sorbonne 17, rue de la Sorbonne 75231 Paris cedex 05

(104) AN, série F 7/4721/5.

(105) Art. XV du décret du 8 brumaire an III (29 octobre 1794) «sur les formalités à remplir pour dénoncer et accuser les représentants du peuple». Archives parlementaires, t. 100, p. 182.

(106) Corinne Gomez-Le Chevanton, «Le procès Carrier. Enjeux politiques, pédagogie collective et construction mémorielle », AHRF, $\mathrm{n}^{\circ} 343$, janvier-mars 2006, p. 76. 